\title{
Neural Correlates of Conscious Self-Regulation of Emotion
}

\author{
Mario Beauregard, ${ }^{1,2,3}$ Johanne Lévesque, ${ }^{3,4}$ and Pierre Bourgouin ${ }^{1}$ \\ 1Département de Radiologie, Faculté de Médecine, Université de Montréal and Centre Hospitalier de l'Université de \\ Montréal (CHUM), Hôpital Notre-Dame, Montréal, Québec, Canada H2L 4M1, ${ }^{2}$ Centre de Recherche en Sciences \\ Neurologiques, Faculté de Médecine, Université de Montréal, Montréal, Québec, Canada H3C 3J7, ${ }^{3}$ Centre de \\ Recherche, Institut Universitaire de Gériatrie de Montréal, Montréal, Québec, Canada H3W 1W5, and “Département de \\ Psychologie, Université de Montréal, Montréal, Québec, Canada H3C 3J7
}

\begin{abstract}
A fundamental question about the relationship between cognition and emotion concerns the neural substrate underlying emotional self-regulation. To address this issue, brain activation was measured in normal male subjects while they either responded in a normal manner to erotic film excerpts or voluntarily attempted to inhibit the sexual arousal induced by viewing erotic stimuli. Results demonstrated that the sexual arousal experienced, in response to the erotic film excerpts, was associated with activation in "limbic" and paralimbic structures, such as the right amygdala, right anterior temporal pole, and hypothalamus. In addition, the attempted inhibition of the sexual arousal generated by viewing the erotic stimuli was asso-
\end{abstract}

Emotional self-regulation refers to the heterogeneous set of processes by which individuals influence, consciously and voluntarily, which emotions they have, when they have them, and how they experience and express these emotions (Gross, 1999). Given the multicomponential nature of emotions-which comprise cognitive, experiential, behavioral, and physiological dimensionsemotional self-regulation may be associated with changes in one or more of these response domains (Gross, 1999). This form of self-regulation involves decreasing, maintaining, or increasing both negative and positive emotions (Masters, 1991; Parrott, 1993; Langston, 1994), by using various cognitive regulatory processes such as rationalization, reappraisal, and suppression (Gross, 1999; Hariri et al., 2000).

Disturbances of normal emotional self-regulation might be a key factor in the genesis of depression and anxiety, both of which may involve a chronic inability to suppress negative emotion (Jackson et al., 2000). Regarding this issue, it has been postulated that impulsive aggression and violence also arise as a consequence of defective regulation of emotional responses (Davidson et al., 2000). Given that the ability to modulate emotions is at the heart of the human experience, it is not surprising that the use of emotional self-regulatory processes constitutes the core of several modern psychotherapeutic approaches.

Despite the immense impact of emotional self-regulation for

Received March 8, 2001; revised June 4, 2001; accepted June 28, 2001.

This work was supported by grants from National Sciences and Engineering Research Council of Canada (NSERC) and Département de Radiologie, Faculté de Médecine, Université de Montréal to M.B. We thank the staff of the Département de Radiologie, Centre Hospitalier de l'Université de Montréal (CHUM), Hôpital Notre-Dame, for their skillful technical assistance.

Correspondence should be addressed to Dr. Mario Beauregard, Centre de Recherche, Institut Universitaire de Gériatrie de Montréal, 4565 Queen Mary Road, Montréal, Québec, Canada, H3W 1W5. E-mail: beauregm@magellan.umontreal.ca. Copyright (C) 2001 Society for Neuroscience $0270-6474 / 01 / 210001-06 \$ 15.00 / 0$ ciated with activation of the right superior frontal gyrus and right anterior cingulate gyrus. No activation was found in limbic areas. These findings reinforce the view that emotional selfregulation is normally implemented by a neural circuit comprising various prefrontal regions and subcortical limbic structures. They also suggest that humans have the capacity to influence the electrochemical dynamics of their brains, by voluntarily changing the nature of the mind processes unfolding in the psychological space.

Key words: emotional self-regulation; consciousness; volition; metacognition; prefrontal cortex; "limbic" structures; functional magnetic resonance imaging

the conduct of human affairs in daily life, few things are known with respect to the neurobiological substrate subserving the psychological processes associated with the volitional modulation of emotional responses. With respect to this question, Nauta (1971) and Tucker et al. (1995) have theorized that the frontal neocortex and limbic/paralimbic structures are components of a frontolimbic network involved in the regulation of emotion. More recently, Davidson and associates (2000) have proposed, on the basis of various evidence from experimental lesion studies in animals and clinical neuropsychological, psychophysiological, and functional brain mapping studies in humans, that emotional self-regulation may be normally implemented by a neural circuit consisting of several regions of the prefrontal cortex (PFC) [e.g., orbitofrontal PFC, dorsolateral PFC, anterior cingulate cortex (ACC)], and of subcortical limbic structures, such as the amygdala and the hypothalamus. The present functional magnetic resonance imaging (fMRI) study was undertaken to test the validity of this view.

\section{MATERIALS AND METHODS}

Subjects. Ten healthy male (Caucasian) volunteers (age range, 20-42; mean age, 23.5) participated in this study. Informed consent was obtained from all subjects.

This article is published in The Journal of Neuroscience, Rapid Communications Section, which publishes brief, peerreviewed papers online, not in print. Rapid Communications are posted online approximately one month earlier than they would appear if printed. They are listed in the Table of Contents of the next open issue of JNeurosci. Cite this article as: JNeurosci, 2001, 21:RC165 (1-6). The publication date is the date of posting online at www.jneurosci.org.

http://www.jneurosci.org/cgi/content/full/5619 
Behavioral procedures. Blood oxygen level-dependent (BOLD) signal changes were measured during two experimental conditions, i.e., a sexual arousal condition and an attempted inhibition condition. In the sexual arousal condition, subjects viewed a series of erotic film excerpts and emotionally neutral film excerpts. They were instructed to react normally to both types of stimuli. In the case of the erotic film excerpts, this meant that the subjects had to allow themselves to become sexually aroused in response to the erotic film excerpts. In the attempted inhibition condition, subjects were instructed to inhibit any emotional reaction to both categories of stimuli. These instructions meant that the subjects had to voluntarily decrease the intensity of the sexual arousal felt in reaction to the erotic film excerpts. To accomplish that goal, subjects were encouraged to distance themselves from these stimuli, that is, to become a detached observer. They were also instructed to look at the stimuli directly during both experimental conditions.

Erotic film excerpts were selected on the basis of previous work by our group demonstrating the efficiency of such stimuli at bringing rapidly and automatically a marked positive change in the subjective emotional experience of healthy male subjects, change correlated with significant activation of limbic brain regions (Beauregard et al., 1998). These film excerpts depicted different kinds of sexual interactions (e.g., oral sex, vaginal intercourse, etc.) between one woman and two or three men, two women and one man, and between two or more women. The emotionally neutral film excerpts were matched to the erotic film excerpts with respect to the number and the gender of the individuals involved in various social interactions (e.g., interviews, carpentry, etc). Erotic film excerpts were presented with their original sound tracks consisting of instrumental music. To avoid any confounding effect attributable to the information processing of language perceived in an auditory manner, emotionally neutral music was superimposed on the neutral film excerpts. Both categories of film excerpts were selected from a private bank of validated stimuli created by the investigators.

The sexual arousal condition corresponded to one functional run and consisted of the viewing of, first, four blocks of emotionally neutral film excerpts and, then, four blocks of erotic film excerpts. On the basis of evidence gathered previously by our group, this design was adopted to avoid contamination of the neutral stimuli by the erotic stimuli. Each block lasted $39 \mathrm{sec}$. Blocks were separated by resting periods of $15 \mathrm{sec}$, during which subjects viewed a blank cyan screen. The attempted inhibition condition corresponded to the other functional run and also consisted of the viewing of, first, four blocks of emotionally neutral film excerpts and, then, four blocks of erotic film excerpts. The order of presentation of the functional runs was counterbalanced across subjects. In addition, the neutral and erotic stimuli that were used in the sexual arousal condition for half of the subjects were presented for the other half of the subjects in the attempted inhibition condition (and vice versa).

To assess the subjective responses of the subjects to the stimuli, immediately at the end of each functional run, they were asked to rate verbally - on a visual analog rating scale ranging from 0 (absence of any emotional reaction) to 8 (strongest emotion ever felt in one's lifetime)the average intensity of sexual arousal or primary emotions [e.g., sadness, happiness, disgust, fear, anger, surprise (Plutchik, 1994)] felt during the viewing of both categories of film excerpts. For each functional run, an average rating score was computed for the four blocks of erotic film excerpts and the four blocks of emotionally neutral film excerpts. At the end of the scanning session, subjects were also asked to complete a "strategy questionnaire" in which they described the emotion regulation strategies they used to inhibit the sexual arousal generated by the erotic stimuli.

MRI. Echoplanar images (EPIs) were acquired on a 1.5 Tesla system (Magnetom Vision, Siemens Electric, Erlangen, Germany). Twentyeight slices ( $5 \mathrm{~mm}$ thick) were acquired every $3 \mathrm{sec}$ in an inclined axial plane, aligned with the anterior commissure-posterior commissure axis. These $\mathrm{T} 2 *$ weighted functional images were acquired using an EPI pulse sequence [repetition time $(\mathrm{TR})=0.8 \mathrm{msec}$; echo time $(\mathrm{TE})=54 \mathrm{msec}$; Flip angle $=90^{\circ}$; field of view $($ FOV $)=215 \mathrm{~mm}$; Matrix $\left.\left.=64 \times 64\right)\right]$. After functional scanning, high-resolution data were acquired via a Tlweighted three-dimensional volume acquisition obtained using a gradient echo pulse sequence $\left(\mathrm{TR}=9.7 \mathrm{msec} ; \mathrm{TE}=4 \mathrm{msec}\right.$; Flip angle $=12^{\circ}$; $\mathrm{FOV}=215 \mathrm{~mm}$; Matrix $=256 \times 256)$.

Data were analyzed using Statistical Parametric Mapping software (SPM99, Wellcome Department of Cognitive Neurology, London, UK). Images for all subjects were realigned to correct for artifacts caused by small head movements and spatially normalized into an MRI stereotactic space (Talairach and Tournoux, 1988). Images were then convolved in space with a three-dimensional isotropic Gaussian kernel (12 $\mathrm{mm}$ full width half maximum) to improve the signal-to-noise ratio and to accommodate for residual variations in functional neuroanatomy that usually persist between subjects after spatial normalization.

For both sexual arousal and attempted inhibition conditions, a conventional subtraction method was used to contrast the brain activity associated with the viewing of the erotic film excerpts and that associated with the viewing of the emotionally neutral film excerpts. For the statistical analysis, the time series of the images were correlated with the delayed boxcar function that approximates the activation patterns, and a fixed effects general linear model for autocorrelated observations was applied voxelwise. Voxel values for each contrast yielded a statistical parametric map of the $t$ statistic (SPM $t$ ), subsequently transformed to the unit normal distribution (SPM Z). An a priori search strategy was used. A whole-brain post hoc analysis was also performed. The a priori search strategy predicted significant activation in the PFC (orbitofrontal PFC, dorsolateral PFC, anterior cingulate cortex), the amygdala, the temporal pole, and the hypothalamus. The search volume corresponding to the brain regions of interest was defined a priori by tracing the neuroanatomical boundaries of these regions on the MR reference image. These boundaries were also traced using the standard atlas of Talairach and Tournoux (1988). For this a priori search, a corrected probability threshold of $p<0.05$ was used. For the whole-brain post hoc analysis, a corrected probability threshold of $p<0.005$ was used. Only clusters showing a spatial extent of at least 10 contiguous voxels were kept for image analysis.

\section{RESULTS}

\section{Behavioral data}

Phenomenologically, the viewing of the erotic film excerpts in both the sexual arousal and attempted inhibition conditions induced a transient state of sexual arousal in all subjects. The mean level of reported sexual arousal was significantly higher in the sexual arousal condition (mean $=5$; range, $3-8$ ) than in the inhibition condition (mean $=2$; range, $1-4)(p<0.005)$. For both experimental conditions, the viewing of the erotic film excerpts did not produce changes in the emotional state other than sexual arousal. Likewise, in both of these conditions, the viewing of the emotionally neutral film excerpts did not generate primary emotional responses. Furthermore, the strategy questionnaire indicated that, in the attempted inhibition condition, most subjects succeeded at distancing themselves from the erotic stimuli, i.e., at becoming a detached observer.

\section{fMRI data}

\section{A priori search}

When the brain activity associated with the viewing of the emotionally neutral film excerpts was subtracted from that associated with the viewing of the erotic film clips in the sexual arousal condition, significant BOLD signal increases were found in the right amygdala, the right anterior temporal pole [Brodmann area (BA) 38], and the hypothalamus (Fig. 1, Table 1). In addition, when the brain activity associated with the viewing of the emotionally neutral film excerpts was subtracted from that associated with the viewing of the erotic film clips in the attempted inhibition condition, significant loci of activation were noted in the right superior frontal gyrus (BA 10) and the right anterior cingulate gyrus (BA 32) (Fig. 2, Table 1). No significant loci of activation were detected in the amygdalas, the anterior temporal polar region, and the hypothalamus (even for a low height threshold value of $p<0.1$, uncorrected). With regard to individual data, the same pattern was found; that is, right prefrontal activation was seen in all 10 subjects, whereas no activation was noted in the amygdala and the hypothalamus, and activation in the anterior temporal pole was noted in only one subject. 


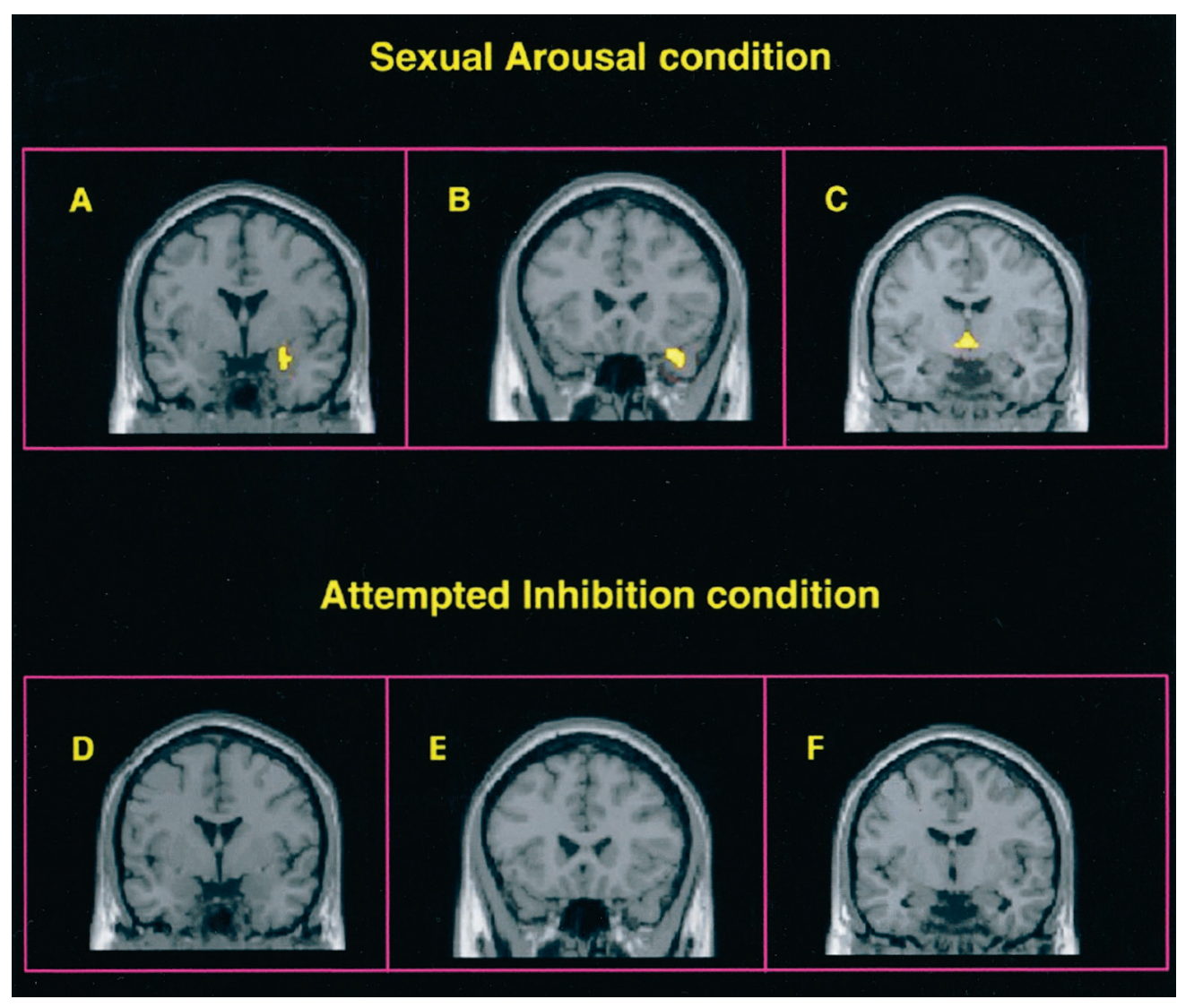

Figure 1. Statistical activation maps are shown for limbic-paralimbic structures defined a priori. Images are coronal sections for the data averaged across subjects. The right hemisphere of the brain corresponds to the right side of the image. In the Sexual Arousal condition, greater activation during the viewing of erotic film excerpts relative to the viewing of emotionally neutral film excerpts was noted in the right amygdala $(A)$, right anterior temporal pole $(B)$, and hypothalamus $(C)$. In the Attempted Inhibition condition, no significant loci of activation were seen in the amygdalas $(D)$, anterior temporal polar region $(E)$, and hypothalamus $(F)$.

Table 1. BOLD signal increases in the search volume defined a priori $(p<0.05$, corrected $)$

Talairach coordinates $(\mathrm{mm})$

\begin{tabular}{|c|c|c|c|c|c|c|}
\hline & \multicolumn{5}{|l|}{ Talairach coordinates $(\mathrm{mm})$} & \multirow[b]{2}{*}{$Z$ statistic } \\
\hline & Region & Brodmann area & $x$ & $\mathrm{y}$ & $\mathrm{z}$ & \\
\hline \multirow[t]{3}{*}{ Sexual arousal condition } & $\mathrm{R}$ amygdala & & 25 & -3 & -14 & 4.17 \\
\hline & $\mathrm{R}$ anterior temporal pole & 38 & 32 & 20 & -26 & 4.05 \\
\hline & Hypothalamus & & 1 & -7 & -9 & 3.97 \\
\hline \multirow[t]{2}{*}{ Attempted inhibition condition } & $\mathrm{R}$ superior prefrontal gyrus & 10 & 22 & 46 & 13 & 4.25 \\
\hline & $\mathrm{R}$ anterior cingulate gyrus & 32 & 9 & 42 & 4 & 3.8 \\
\hline
\end{tabular}

Stereotaxic coordinates are derived from the human atlas of Talairach and Tournoux (1988) and refer to medial-lateral position $(x)$ relative to medline (positive $=$ right) anterior-posterior position $(y)$ relative to the anterior commissure (positive $=$ anterior), and superior-inferior position $(z)$ relative to the commissural line (positive $=$ superior). Designation of Brodmann areas for cortical areas are also based on this atlas. L, Left; R, right.

\section{Whole-brain post hoc analysis}

When the brain activity associated with the viewing of the emotionally neutral film excerpts was subtracted from that associated with the viewing of the erotic film clips in the sexual arousal condition, significant BOLD signal increases were seen in the left middle occipital gyrus (BA 19), the right superior occipital gyrus (BA 19), the right inferior temporal gyrus (BA 37), the left cerebellum, and the superior parietal lobule (BA 7), bilaterally (Table 2). Moreover, when the brain activity associated with the viewing of the emotionally neutral film excerpts was subtracted from that associated with the viewing of the erotic film clips in the attempted inhibition condition, significant loci of activation were found in the left middle occipital gyrus (BA 19), the right inferior and superior occipital gyri (BA 19), the left superior occipital gyrus (BA 19), the left inferior frontal gyrus (BA 44), and the superior parietal lobule (BA 7), bilaterally (Table 2).

\section{DISCUSSION}

In agreement with the results of a previous study by our group (Karama et al., 2001), the viewing of erotic film excerpts, in the sexual arousal condition, produced a significant activation of the right amygdala, right anterior temporal pole (BA 38), and hypothalamus. These findings fit with the various lines of evidence suggesting that the amygdala plays a pivotal role in the evaluation of the emotional significance of stimuli (for review, see Lane and Nadel, 2000), that the hypothalamus is a key brain structure implicated in the neuroendocrine and autonomic expression of emotion (Carter, 1998), and that the anterior temporal pole is involved in the imparting of emotional color to subjective experience (Mesulam, 1985). In light of the anatomic projections that the central nucleus of the amygdala sends to the hypothalamus, and the basolateral nuclei of the amygdala sends to the temporal pole (Martin, 1996), it appears conceivable that in the sexual arousal condition, the right amygdala, the hypothalamus, and the 
Figure 2. Statistical activation maps showing peaks of activation in prefrontal cortical regions defined a priori during the Attempted Inhibition condition. Images are coronal sections for the data averaged across subjects. The right hemisphere of the brain corresponds to the right side of the image. Significant loci of activation were noted in the right superior frontal gyrus $(A)$ and right anterior cingulate gyrus $(B)$.

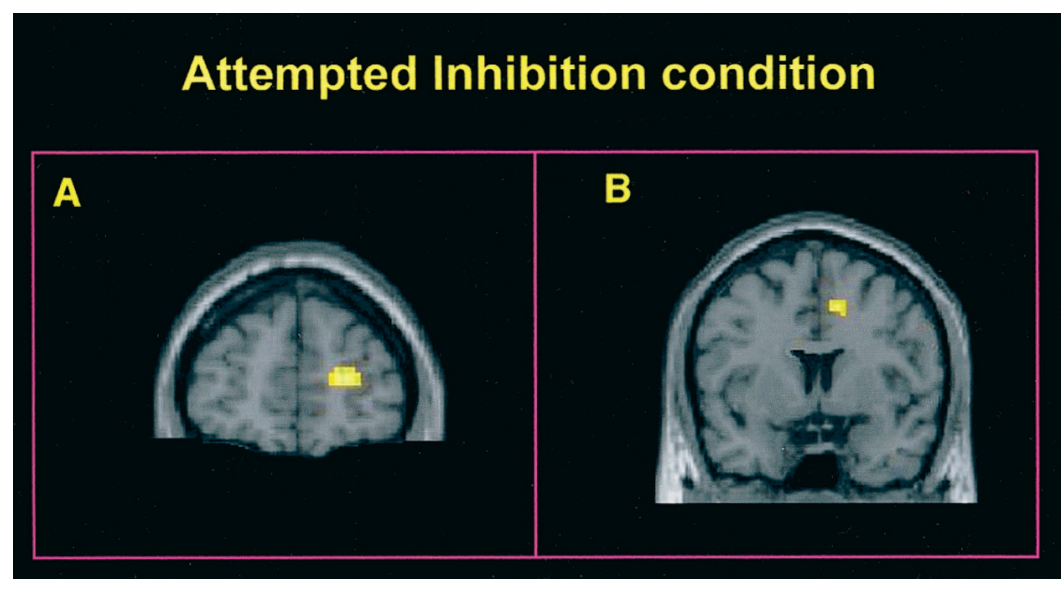

Table 2. BOLD signal increases in the whole-brain post hoc analysis $(p<0.005$, corrected)

Talairach coordinates $(\mathrm{mm})$

\begin{tabular}{|c|c|c|c|c|c|c|}
\hline & & & & & & \multirow[b]{2}{*}{$Z$ statistic } \\
\hline & Region & $\begin{array}{l}\text { Brodmann } \\
\text { area }\end{array}$ & $x$ & $\mathrm{y}$ & $\mathrm{z}$ & \\
\hline \multirow[t]{6}{*}{ Sexual arousal condition } & L middle occipital gyrus & 19 & -48 & -72 & 0 & 19.2 \\
\hline & $\mathrm{R}$ inferior temporal gyrus & 37 & 51 & -66 & -9 & 17.48 \\
\hline & R superior occipital gyrus & 19 & 30 & -70 & 33 & 11.9 \\
\hline & $\mathrm{R}$ superior parietal lobule & 7 & 27 & -57 & 60 & 11.85 \\
\hline & L superior parietal lobule & 7 & -30 & -54 & 60 & 11.83 \\
\hline & L cerebellum & & -24 & -84 & 21 & 11.28 \\
\hline \multirow[t]{7}{*}{ Attempted inhibition condition } & L middle occipital gyrus & 19 & -48 & -72 & -3 & 13.71 \\
\hline & $\mathrm{R}$ inferior occipital gyrus & 19 & 45 & -72 & 3 & 11.01 \\
\hline & $\mathrm{R}$ superior occipital gyrus & 19 & 30 & -78 & 36 & 10.11 \\
\hline & L superior parietal lobule & 7 & -27 & -54 & 54 & 9.49 \\
\hline & L superior occipital gyrus & 19 & -21 & -84 & 21 & 9.38 \\
\hline & $\mathrm{R}$ superior parietal lobule & 7 & 30 & -54 & 60 & 7.68 \\
\hline & $\mathrm{L}$ inferior frontal gyrus & 44 & -57 & 9 & 30 & 5.67 \\
\hline
\end{tabular}

L, Left; R, right.

right anterior temporal pole are functional components of a neural circuit subserving the processing of the erotic visual stimuli. Within such a network, we surmise that, first, the right amygdala participated in the evaluation of the emotional content of the complex perceptual information associated with the visual processing of the erotic film excerpts. Second, this brain structure sent a message to the hypothalamus. Such modulation of the hypothalamic activity then led to sexual arousal (Sachs and Meisel, 1984). Third, for this physiological arousal to become fully conscious, in addition to sending outputs to the hypothalamus, the amygdala sent, in parallel, a message to the right anterior temporal pole. Such a role for this brain region concurs with recent work showing that this paralimbic associative cortical area is correlated with attending to one's own emotional experience (Lane, 2000).

The results of the attempted inhibition condition revealed that the volitional inhibitory action produced by the male subjects, to decrease the intensity of the sexual arousal produced by the erotic film excerpts, was associated with activation peaks in BA 10 of the right dorsolateral PFC (superior frontal gyrus) and BA 32 of the right ACC. Contrary to the pattern of brain activity seen in the sexual arousal condition, in the attempted inhibition condition, no significant loci of activation were noted in the amygdalas, the anterior temporal polar region, and the hypothalamus (even for a low height threshold value of $p<0.1$, uncorrected). Furthermore, the analysis of individual data revealed the same pattern, i.e., no significant loci of activation was found in the amygdala, the anterior temporal polar region, and the hypothalamus whenever the right superior frontal gyrus and right anterior cingulate gyrus were activated. These findings provide some support for the hypothesis that the dorsolateral PFC, the amygdala, and the hypothalamus participate in a neural circuit involved in emotional self-regulation (Davidson et al., 2000). They are also consistent with the results of a recent functional brain imaging study showing that a cognitive task, such as choosing labels that identify the emotional expression of faces, correlated with simultaneous increased regional cerebral blood flow (rCBF) in the right prefrontal cortex and decreased $\mathrm{rCBF}$ in the amygdalas (Hariri et al., 2000). In other respects, these results are consistent with findings indicating that the dorsolateral PFC is implicated in metacognitive/executive top-down processes, which refer to the ability to monitor and control the information processing necessary to produce voluntary action (Flavell, 1979). These results also accord with the view that, by virtue of its anatomic connections with brain regions implicated in the modulation of autonomic and endocrine functions, such as the amygdala, hypothalamus, and orbitofrontal PFC, the rostral-ventral affective subdivision of the ACC is primarily involved in the 
evaluation of emotional and motivational information and in the regulation of emotional responses (Vogt et al., 1992; Devinsky et al., 1995; Bush et al., 2000).

The whole-brain post hoc analysis revealed that in addition to producing activation in the right amygdala, right anterior temporal pole, and hypothalamus, the sexual arousal condition also induced significant activation in the inferotemporal cortex, the extrastriate visual cortex, the cerebellum, and the superior parietal lobule. Moreover, in addition to producing activation in the right superior frontal gyrus and the right anterior cingulate gyrus, the attempted inhibition condition was also associated with activation in the extrastriate visual cortex, the superior parietal lobule, and the left inferior frontal gyrus.

Clinical neuropsychological data have demonstrated that cerebellar lesions can produce a blunting of effect (Schmahmann and Sherman, 1998). In light of these data, and on the basis of the fact that cerebellar activation has often been noted in functional brain imaging studies of emotion generated by emotionally laden visual stimuli, one could argue that the cerebellar activation found here suggests that the cerebellum is somehow related to the "feeling" experience associated with sexual arousal. Further studies are awaited to tackle this intriguing question.

With respect to the inferotemporal cortex, the results of a recent magnetoencephalography study (Streit et al., 1999) suggest that this cortical region is involved in the recognition of the various facial expressions of emotions. Given these findings and the nature of the erotic film excerpts used in this study, it seems plausible that the inferotemporal activation seen here may have something to do with such a function.

The activation of the extrastriate visual cortex is in keeping with the results of several functional neuroimaging studies having shown that, when compared with neutral visual stimuli, emotionally laden visual stimuli elicit increased activation in this brain area (Lane et al., 1997, 1999; Reiman et al., 1997; Beauregard et al., 1998; Lang et al., 1998). Assuming that viewing erotic stimuli automatically leads to increased attentional tapping, the extrastriate cortical activation noted here is consistent with the hypothesis that attention to visual stimuli can modulate neural activity in the extrastriate visual cortex (Corbetta et al., 1991; O'Craven et al., 1997; Büchel et al., 1998; Chawla et al., 1999). With respect to this issue, the results of a recent positron emission tomography study (Lane et al., 1999) suggest that the amygdala may exert modulatory effects in extrastriate visual cortex during visual processing of highly arousing emotional stimuli. Such a neuromodulatory action could constitute an evolutionary beneficial trait permitting more efficient attention to stimuli having a strong survival value. Along the same lines, the activation noted here in the superior parietal lobule, a brain region implicated in sustained attention to emotionally neutral stimuli (Le et al., 1998), might derive from the impact of emotional arousal on attentional mechanisms that are grounded neurally in this area of the parietal lobe.

The left inferior frontal activation seen in the attempted inhibition condition was localized in a portion of Broca's area (BA 44). Functional brain imaging techniques have shown that this cortical region is part of a neural circuit implementing the subvocal rehearsal component of verbal working memory (Smith and Jonides, 1998). In the present context, it is possible that the activation noted in this prefrontal cortical area was related to the internal speech generated by the subjects during their attempt to inhibit the sexual arousal associated with the visual processing of the erotic film excerpts.

The normal functioning of the neural network linking the right dorsolateral PFC, right ACC, right amygdala, right anterior temporal pole, and hypothalamus may constitute a fundamental psychobiological mechanism through which human beings can consciously and willfully self-regulate their emotional responses, using various metacognitive processes. From a phylogenetic perspective, such a circuit may implement one of the most remarkable human faculties that has emerged in the course of human evolution. At both an individual and a collective level, a defect of this neural circuitry (or of an analogous network involving a prefrontal cortical modulation of negative emotional responses associated with activation in limbic structures) may have disastrous psychological and social consequences. Ontologically, the present findings suggest that humans have the capacity to influence the electrochemical dynamics of their brains, by voluntarily changing the nature of the mind processes unfolding in the psychological space.

Last, we acknowledge some of the limitations that characterize the present study. First, the restriction to one arousal state makes it difficult to argue for the specificity, or generality, of the brain findings for both the sexual arousal and attempted inhibition conditions. Second, because there was no experimental condition in which subjects had to voluntarily increase their arousal state, it remains possible that the activations seen in the attempted inhibition condition reflected an effort to modulate the sexual arousal induced by the erotic stimuli rather than an actual inhibition of this arousal.

\section{REFERENCES}

Beauregard M, Leroux J-M, Bergman S, Arzoumanian Y, Beaudoin G, Bourgouin P, Stip E (1998) The functional neuroanatomy of major depression: an fMRI study using an emotional activation paradigm. NeuroReport 9:3253-3258.

Büchel C, Josephs O, Rees G, Turner R, Frith CD, Friston KJ (1998) The functional anatomy of attention to visual motion: a functional MRI study. Brain 121:1281-1294.

Bush G, Luu P, Posner MI (2000) Cognitive and emotional influences in anterior cingulate cortex. Trends Cogn Sci 4:215-222.

Carter S (1998) Neuroendocrine perspectives on social attachment and love. Psychoneuroendocrinology 23:779-818.

Chawla D, Rees G, Friston KJ (1999) The physiological basis of attentional modulation in extrastriate visual areas. Nat Neurosci 2:671-676.

Corbetta M, Miezin FM, Dobmeyer S, Shulman GL, Petersen SE (1991) Selective and divided attention during visual discriminations of shape color, and speed: functional anatomy by positron emission tomography. J Neurosci 11:2383-2402.

Davidson RJ, Putnam KM, Larson CL (2000) Dysfunction in the neural circuitry of emotion regulation: a possible prelude to violence. Science 289:591-594.

Devinsky O, Morrell MJ, Vogt BA (1995) Contributions of anterior cingulate cortex to behaviour. Brain 118:279-306.

Flavell JH (1979) Metacognition and cognitive monitoring: a new area of cognitive development inquiry. Am Psychol 34:906-911.

Gross JJ (1999) Emotion regulation: past, present, future. Cogn Emotion 13:551-573.

Hariri A, Bookheimer SY, Mazziotta JC (2000) Modulating emotional responses: effects of a neocortical network on the limbic system. NeuroReport 11:43-48.

Jackson DC, Malmstadt JR, Larson CL, Davidson RJ (2000) Suppression and enhancement of emotional responses to unpleasant pictures. Psychophysiology 37:515-522.

Karama S, Lecours AR, Leroux J-M, Bourgouin P, Beaudoin S, Beauregard M 2001 Areas of brain activation in males and females during viewing of erotic film excerpts. Hum Brain Mapp, in press.

Lane RD (2000) Neural correlates of conscious emotional experience. In: Cognitive neuroscience of emotion (Lane RD, Nadel L, eds), pp 345-370. New York: Oxford UP

Lane RD, Nadel L (2000) Cognitive neuroscience of emotion. New York: Oxford UP

Lane RD, Reiman EM, Bradley MM, Lang PJ, Ahern GL, Davidson RJ, Schwartz GE (1997) Neuroanatomical correlates of pleasant and unpleasant emotion. Neuropsychologia 35:1437-1444.

Lane RD, Chua PM-L, Dolan RJ (1999) Common effects of emotional valence, arousal and attention on neural activation during visual processing of pictures. Neuropsychologia 37:989-997. 
Lang PJ, Bradley MM, Fitzsimmons JR, Cuthbert BN, Scott JD, Moulder B, Nangia V (1998) Emotional arousal and activation of the visual cortex: an fMRI analysis. Psychophysiology 35:199-210.

Langston CA (1994) Capitalizing on and coping with daily-life events: expressive responses to positive events. J Pers Soc Psychol 67:1112-1125.

Le TH, Pardo JV, Hu X (1998) 4-T-fMRI study of nonspatial shifting of selective attention: cerebellar and parietal contributions. J Neurophysiol 79:1535-1548.

Martin JH (1996) Neuroanatomy. Stanford: Appleton \& Lange.

Masters JC (1991) Strategies and mechanisms for the personal and social control of emotion. In: The development of emotion regulation and dysregulation (Garber J, Dodge KA, eds), pp 182-207. Cambridge, UK: Cambridge UP.

Mesulam M-M (1985) Patterns in behavioural neuroanatomy: Association areas, the limbic system, and hemispheric specialization. In: Principles of behavioral neurology (Mesulam M-M, ed), pp 1-70. Philadelphia: F. A. Davis Company.

Nauta WJH (1971) The problem of the frontal lobe: a reinterpretation. J Psychiatr Res 8:167-187.

O’Craven KM, Rosen BR, Kwong KK, Treisman A, Savoy RL (1997) Voluntary attention modulates fMRI activity in human MT-MST. Neuron 18:591-598.

Parrott WG (1993) Beyond hedonism: motives for inhibiting good moods and for maintaining bad moods. In: Handbook of mental control
(Wegner DM, Pennebaker JW, eds), pp 278-308. Englewood Cliffs, NJ: Prentice-Hall.

Plutchik R (1994) The psychology and biology of emotion. New York: Harper Collins.

Reiman EM, Lane RD, Ahern GL, Schwartz GE, Davidson RJ, Friston KJ, Yun L-S, Chen K (1997) Neuroanatomical correlates of externally and internally generated human emotion. Am J Psychiatry 154:918-925.

Sachs BD, Meisel RL (1984) The physiology of male sexual behavior. In: Physiology of reproduction (Knobil E, Neill JD, eds), pp 3-105. New York: Raven.

Schmahmann JD, Sherman JC (1998) The cerebellar cognitive affective syndrome. Brain 121:561-579.

Smith EE, Jonides J (1998) Neuroimaging analyses of human working memory. Proc Natl Acad Sci USA 95:12061-12068.

Streit M, Ionnides AA, Liu L, Wolver W, Dammers J, Gross J, Gaebel W, Muller-Gartner HW (1999) Neurophysiological correlates of the recognition of facial expressions of emotion as revealed by magnetoencephalography. Brain Res Cogn Brain Res 7:481-491.

Talairach J, Tournoux P (1988) Co-planar stereotaxic atlas of the human brain. Stuttgart: Thieme.

Tucker DM, Luu P, Pribram KH (1995) Social and emotional selfregulation. Ann NY Acad Sci 769:213-239.

Vogt BA, Finch DM, Olson CR (1992) Functional heterogeneity in cingulate cortex: the anterior executive and posterior evaluative regions. Cereb Cortex 2:435-443. 